\title{
721 INTRATUMORAL IMMUNOTHERAPY WITH ALUMINUM HYDROXIDE-TETHERED IL-12 INDUCES POTENT LOCAL AND SYSTEMIC IMMUNITY WITH MINIMAL TOXICITY
}

${ }^{1}$ Michael Schmidt*, 'Gregory Papastoitsis, ${ }^{1}$ Howard Kaufman, ${ }^{2}$ Darrell Irvine, ${ }^{2} \mathrm{~K}$ Wittrup. ${ }^{1}$ Ankyra Therapeutics, Boston, MA, United States; ${ }^{2}$ Massachusetts Institute of Technology, Cambridge, MA, United States

Background Interleukin-12 (IL-12) is a potent pro-inflammatory cytokine that promotes Th1 skewing, IFN $\gamma$ expression, Tand NK-cell activation, and antigen presentation. In animal models, IL-12 can elicit robust anti-tumor responses through activation of both innate and adaptive immunity. However, clinical translation of IL-12 has been hindered by significant immune-related toxicity when delivered systemically, necessitating low doses that are often insufficient for efficacy. Intratumoral (IT) administration can expand the therapeutic window of IL-12 by increasing the local tumor concentration relative to systemic exposure but is in turn limited by rapid vascular and lymphatic clearance of injected drug from the tumor and corresponding systemic accumulation. Here we describe an approach to locally retain intratumorally administered IL-12 by complexing it to the common vaccine adjuvant aluminum hydroxide (alum) through a novel phosphopeptide linkage.

Methods Single-chain murine IL-12 (mIL12) was genetically fused at its c-terminus to a short alum-binding peptide (ABP) that is specifically phosphorylated on multiple serines when co-expressed with the kinase Fam20C. Phosphorylated mIL12$\mathrm{ABP}$ proteins were complexed with a $10 \mathrm{x}$ mass excess of aluminum hydroxide through a naturally occurring ligand exchange reaction between the phosphoserines in the ABP and surface hydroxyl groups on alum. mIL12-ABP/alum complexes were characterized for in vitro potency and in vivo efficacy in multiple syngeneic tumor models including MC38, CT26, A20, 4T1, and B16F10 following IT administration. Immune analyses and re-challenge experiments are in progress.

Results mIL12-ABP is phosphorylated on multiple sites when co-expressed with Fam20C and is stably retained on aluminum hydroxide in vitro under elution conditions containing phosphate and serum. Alum-bound mIL12-ABP remains active in cellular assays with a 3-4 fold increase in EC50 compared to free protein. Following intratumoral administration, the mIL12-ABP/alum complexes have significantly extended tumor retention compared to unmodified mIL12, leading to potent local immune activation for $>1$ week. One or two doses of IT administered mIL12-ABP/alum is sufficient to induce robust monotherapy efficacy in diverse syngeneic tumor models including cold tumors resistant to checkpoint blockade and other immunotherapies. Locally administered mIL12-ABP/alum is further able to prime a systemic immune response leading to efficacy against non-injected tumors and spontaneous metastases. Doses required for optimal efficacy are well tolerated in mice with no significant weight loss or other evidence of systemic toxicity.

Conclusions Ankyra's platform is a differentiated approach to expand the therapeutic window of IL-12 and other cytokine drugs by enhancing tumor retention following IT administration.

http://dx.doi.org/10.1136/jitc-2021-SITC2021.721 